\title{
Rescue of hemodynamic compromise in superior vena cava syndrome with transesophageal echocardiography
}

\author{
Il-Seok Kim, Ho Kyun Shin, Sang-Soo Kang, Joon-Hee Park, and Chang-Geun Song \\ Department of Anesthesiology and Pain Medicine, Kangdong Sacred Heart Hospital, Hallym University College of Medicine, Seoul, Korea
}

The anesthetic management of patients with an anterior mediastinal mass is very challenging due to the potential risk of hemodynamic and respiratory compromise. A specialized anesthetic plan should be developed for in patients with an anterior mediastinal mass based on the preoperative clinical symptoms as well as, radiographic, computed tomographic, spirometric, and echocardiographic data [1,2].

A 31-year-old man (height $173.5 \mathrm{~cm}$, weight $70.4 \mathrm{~kg}$ ) came to our emergency department with right-sided chest discomfort, dry cough, progressive dyspnea, and orthopnea for the previous 4 weeks. The initial chest X-ray evaluation revealed a large amount of right side pleural effusion and a bulky mediastinal mass. Multi directional computed tomography revealed a 12.3 $\mathrm{cm}$ homogenous soft tissue mass in the anterior mediastinum compressing the superior vena cava and displacing the ascending aorta. The trachea and both main bronchi were patent, but a cross-sectional area of the right upper lobe bronchus $(7.21 \mathrm{~mm})$ was reduced $44 \%$ relative to the left upper lobe bronchus (12.97 $\mathrm{mm}$ ). Right loculated pleural effusion, totally collapsed right lower lobe and subsegmental atelectasis in the right middle lobe were noted. A therapeutic and diagnostic thoracentesis was done under local anesthesia, and 1,600 $\mathrm{ml}$ of pleural fluid was removed. Cytology of pleural fluid revealed many lymphocytes and mesothelial cells, but these were negative for malignancy. However, because dyspnea persisted and pleural effusion was increased after admission, a mediastinoscopic biopsy and pleural decortication were performed under general anesthesia. In the operating room, the CAPIOX emergency bypass system
(CAPIOX $^{\circledR}$ EBS $^{\mathrm{TM}}$, Terumo Corporation, Tokyo, Japan) was prepared in the event of cardiopulmonary collapse. Before the induction of general anesthesia, a 20-guage catheter was placed in the right radial artery for continuous blood pressure monitoring. The patient was placed in the Semi-Fowler's position with the head elevated 30 degrees to relieve the discomfort of the supine position. Initial vital signs were blood pressure 105/65 $\mathrm{mmHg}$, heart rate 100 beats/min, respiration 25 breaths/min, and $\mathrm{O}_{2}$ saturation $96 \%$. Preoxygenation with $100 \% \mathrm{O}_{2}$ was administered using a face mask, and anesthesia was induced with a continuous infusion of propofol (effect-site concentration 4.0-5.0 $\mu \mathrm{g} / \mathrm{ml}$ and remifentanil (effect- site concentration 3.0$5.0 \mathrm{ng} / \mathrm{ml}$ using a target-controlled infusion pump (Orchestra ${ }^{\circledR}$ Base Primea, Fresenius Vial, Brezins, France) and a bolus dose of rocuronium $50 \mathrm{mg}$ via a preexisting 16-guage IV catheter in the vein on the medial surface of the right leg. Intubation was successfully achieved using the left-sided Robert-Shaw double lumen bronchial tube in the supine position. Breath sounds were heard on both sides of the chest. Mechanical ventilation was maintained with a tidal volume, respiratory rate and partial pressure of end-tidal carbon dioxide of $500 \mathrm{ml}, 15$ breaths/min, and $28-32 \mathrm{mmHg}$, respectively.

After induction, the arterial blood pressure began to drop to $60 / 35 \mathrm{mmHg}$ and the heart rate increased to 110 beats $/ \mathrm{min}$. Ephedrine $10 \mathrm{mg}$ were administered intravenously, but the vital signs were not improved. A transesophageal echocardiography (TEE) was performed for evaluation of hemodynamic instability. It showed that a large mass compressed the right atrium

Corresponding author: Il-Seok Kim, M.D., Department of Anesthesiology and Pain Medicine, Kangdong Sacred Heart Hospital, Hallym University College of Medicine, 445, Gil-dong, Gangdong-gu, Seoul 134-010, Korea. Tel: 82-2-2224-2659, Fax: 82-2-474-0956, E-mail: gns70@ hallym.or.kr

(ㄷ) This is an open-access article distributed under the terms of the Creative Commons Attribution Non-Commercial License (http:// creativecommons.org/licenses/by-nc/3.0/), which permits unrestricted non-commercial use, distribution, and reproduction in any medium, provided the original work is properly cited. 

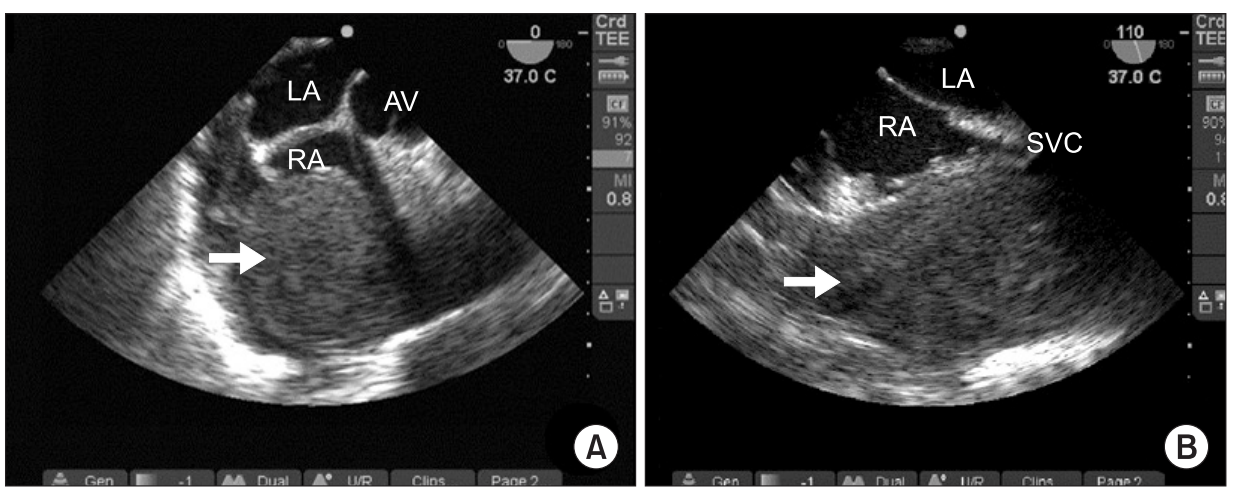

Fig. 1. Transesophageal echocardiographic, midesophageal modified aortic valve short-axis view (A) and bicaval view (B). A large mass compresses the right atrium (arrow in $\mathrm{A}$ ) and superior vena cava (arrow in B). AV: aortic valve, LA: left atrium, RA: right atrium, SVC: superior vena cava.

in a midesophageal modified aortic valve short-axis view and also compressed the superior vena cava in a midesophageal bicaval view (Fig. 1). In a transgastric mid-short-axis view and long-axis view, the left ventricle contracted vigorously with reduced end-diastolic and end-systolic area, resulting in severe hypovolemia. The patient was moved to the Trendelenburg position to increase venous return instead of the comfortable preoperative semi-Fowler's position. We accelerated the infusion rate of a lactated Ringer's solution. A central venous catheter was inserted via the left femoral vein with ultrasound guidance and $500 \mathrm{ml}$ of a $6 \%$ hydroxyethyl starch (Voluven ${ }^{\circledR}$, Fresenius Kabi, Homburg, Germany) was infused over 10 minutes. After volume resuscitation, the arterial blood pressure increased to 110/65 mmHg with a heart rate of 95 beats/min.

No further deterioration of the vital signs was noticed and planned surgery proceeded. The patient was placed in the left lateral decubitus position for the mediastinoscopic biopsy and the right pleural decortications under one-lung ventilation. The total operation time was 60 minutes without any events, and blood loss during surgery was estimated to be $100 \mathrm{ml}$, with a urine output of $50 \mathrm{ml}$. The total amount of fluid administered was $1,700 \mathrm{ml}$, including $1,100 \mathrm{ml}$ of lactated Ringer's solution and $600 \mathrm{ml}$ of $6 \%$ hydroxyethyl starch. The bronchial tube was removed after observing full recovery of spontaneous ventilation and muscle power tone of extremities and the patient was transferred to the intensive care unit (ICU) with $\mathrm{O}_{2}$ $5 \mathrm{~L} / \mathrm{min}$ administered through a Venturi mask. In the ICU, no cardiorespiratory abnormality was found except for a reduced urine output of 20-30 ml/hr. The pathologic examination showed T-cell type lymphoblastic lymphoma and the patient was referred to an oncologist for chemotherapy.

TEE is the most powerful diagnostic technique for providing both qualitative and quantitative indices of ventricular systolic function and diastolic function and a rapid qualitative assessment of intra-ventricular volume status. TEE may be used for non-cardiac surgical patients when the patient has a known or suspected cardiovascular pathology that might result in hemodynamic, pulmonary, or neurologic compromise [3]. Furthermore, TEE should be used when unexplained life-threatening circulatory instability persists despite corrective therapy. TEE allows the anesthesiologist to make accurate diagnoses and helps guide clinical decision-making with a more beneficial impact compared with traditional hemodynamic monitoring techniques.

During general anesthesia, a comfortable preoperative position that alleviates the pressure of the mediastinal mass on surrounding structures is recommended [4]. However, as we placed the patient in the preoperative semi-Fowler's position without evaluation for hemodynamic instability using TEE, the patient deteriorated drastically and was predisposed to circulatory collapse.

In conclusion, we experienced a case of hemodynamic compromise associated with superior vena cava syndrome. We diagnosed promptly with TEE, which monitored the degree of right atrium compression as well as the volume status, and treated successfully with positional change and fluid loading. A comprehensive understanding of the pathophysiology, appropriate preoperative preparation, and a powerful diagnostic technique such as TEE may result in safer anesthetic management. 


\section{References}

1. Blank RS, de Souza DG. Anesthetic management of patients with an anterior mediastinal mass: continuing professional development. Can J Anaesth 2011; 58: 853-67.

2. Erdös G, Tzanova I. Perioperative anaesthetic management of mediastinal mass in adults. Eur J Anaesthesiol 2009; $26: 627-32$.

3. Practice guidelines for perioperative transesophageal echocardiography. An updated report by the American Society of Anesthesiologists and the Society of Cardiovascular Anesthesiologists Task Force on Transesophageal Echocardiography. Anesthesiology 2010; 112: 1084-96.

4. Choi WJ, Kim YH, Mok JM, Choi SI, Kim HS. Patient repositioning and the amelioration of airway obstruction by an anterior mediastinal tumor during general anesthesia -A case report-. Korean J Anesthesiol 2010; 59: 206-9. 